\title{
Intra-operative events and countermeasures during esophagectomy via transcervical incision inflatable single-port mediastinoscope combined with laparoscopy
}

\author{
Chengdong Liu", Zihao Chen", Rongqiang Wei", Kenan Huang, Bin Wu, Zhifei Xu, Yunhao Fang, \\ Xinyu Ding, Hua Tang
}

Department of Thoracic Surgery, Shanghai Changzheng Hospital Affiliated to Second Military Medical University, Shanghai, China

Contributions: (I) Conception and design: X Ding, H Tang; (II) Administrative support: Z Xu; (III) Provision of study materials or patients: B Wu; (IV) Collection and assembly of data: C Liu, K Huang, Y Fang; (V) Data analysis and interpretation: C Liu, Z Chen, R Wei; (VI) Manuscript writing: All authors; (VII) Final approval of manuscript: All authors.

\#These authors contributed equally to this work.

Correspondence to: Hua Tang; Xinyu Ding. Department of Thoracic Surgery, Shanghai Changzheng Hospital Affiliated to Second Military Medical University, 415 Fengyang Road, Huangpu District, Shanghai 200003, China. Email: tangh_mits@163.com; dingxy269@163.com.

Background: Esophagectomy via transcervical incision inflatable single-port mediastinoscope combined with laparoscopy as a safe and feasible minimally invasive technique has gained attention recently. But the occurrence of Intraoperative events is inevitable. It's necessary to investigate and discuss the intraoperative events and countermeasures during operation.

Methods: Intraoperative events were retrospectively reviewed in 60 patients who underwent esophagectomy via transcervical incision inflatable single-port mediastinoscope combined with laparoscopy in the recent 3 years.

Results: There was no perioperative death and no aortic or bronchial injury. Bronchial artery injury occurred in 2 cases (3.34\%), bronchial artery combined with azygos vein hemorrhage occurred in 1 case (1.67\%). The pleura were injured in 3 cases (5\%). Recurrent laryngeal nerve injury was noticed in 7 cases (11.67\%). Thoracic duct injury occurred in 1 case (1.67\%).

Conclusions: As a new surgical method, esophagectomy via transcervical incision inflatable single-port mediastinoscope combined with laparoscopy is considered safe and feasible, but requires improvement when compared with traditional surgical methods. Due to the influence of surgical space and with experienced surgeons, the incidence of intraoperative events such as intraoperative bleeding and thoracic duct injury is not dominant when compared with the traditional surgical methods. Thoracic surgeons should continuously improve their clinical knowledge as well as skills. Careful preoperative examination and evaluation of the patients, being familiar with the anatomical structure and various methods, wise selection of energy devices and calmly dealing with all kinds of events are the key factors for successful surgeries with fewer intraoperative events.

Keywords: Esophageal neoplasm; radical resection; inflatable mediastinoscope; intraoperative events

Submitted Jun 29, 2020. Accepted for publication Oct 28, 2020.

doi: $10.21037 /$ jtd-20-2331

View this article at: http://dx.doi.org/10.21037/jtd-20-2331 


\section{Introduction}

Esophageal cancer is one of the main diseases of thoracic cavity, and its incidence of esophageal cancer in China is strikingly high (1), ranking fifth in the incidence of malignant tumors in China $(1,2)$. To date, surgical resection still remains to be the main treatment for early-stage esophageal cancer, and the most common approaches include classical McKeown, Ivor Lewis, videoassisted thoracoscopy combined with laparoscopy, and mediastinoscopy combined with laparoscopy. Among these techniques, esophagectomy via transcervical incision inflatable single-port mediastinoscope combined with laparoscopy as a minimally invasive technique has gained attention recently, especially in patients with dense thoracic adhesion and poor pulmonary function, and who are unable to tolerate the traditional surgical methods $(3,4)$. This operation is performed by transcervical incision under mediastinoscopy without injuring the thorax, and this not only reduces postoperative pain and perioperative cardiopulmonary complications, but also completes total mesentery esophagectomy and adequate lymphadenectomy (5). This technique was first performed by Fujiwara from Japan in $2015(6,7)$, and then was introduced into China in 2016. Today, it has become one of the most compelling research topics in the surgical methods of esophageal cancer. In this paper, the intraoperative surgical events and their management in 60 patients undergoing esophagectomy via transcervical incision inflatable single-port mediastinoscope combined with laparoscopy were summarized in our department from March 2017 to January 2020.

We present the following article in accordance with the STROBE reporting checklist (available at http://dx.doi. org/10.21037/jtd-20-2331).

\section{Methods}

\section{Clinical data}

Since 2016, 60 cases, including 50 males and 10 females, who underwent esophagectomy via transcervical incision inflatable single-port mediastinoscope combined with laparoscopy in our department were enrolled. The patients included were 50 to 82 years old, with a median age of 64.9 years. Fiberoptic esophagoscopy was performed routinely before operation, and adenocarcinoma was found in 2 cases and squamous cell carcinoma was found in 58 cases. Among the 60 patients, there were 6 cases with upper esophageal carcinoma, 36 cases with middle esophageal carcinoma and 18 cases with lower esophageal cancer. Thirty-one cases had T1 stage, 16 cases had T2 stage, and 13 cases had T3 stage according to the TNM staging. All operations were completed successfully, and no intraoperative deaths were observed.

Inclusion criteria: patients with T3N1M0 and below stage; cardiopulmonary function should withstand the anesthetic requirements of routine surgery; no absolute contraindications to surgery; and preoperative pathological diagnosis of esophageal cancer.

Exclusion criteria: patients above T3N1M0 stage; with severe organ dysfunction such as heart, lung, liver, kidney, etc.; with absolute surgical contraindications; and lack of preoperative pathological diagnosis.

\section{Surgical methods}

The patient was placed in supine position (Figure 1), with the head extended backwards and tilted to the right, and draping and covering the neck, chest and abdomen under general anesthesia and double-lumen endotracheal intubation. Two groups of surgeons work simultaneously for performing mediastinoscopy and laparoscopy. In the neck, a $4 \mathrm{~cm}$ longitudinal incision was made along the medial edge of the sternocleidomastoid muscle, and dissected into the esophagus along the medial side of the sternocleidomastoid muscle, freeing the encircling and marking of the esophagus with an elastic loop to protect the recurrent laryngeal nerve, and then the protective cover of $5 \mathrm{~cm}$ incision was placed. During their practice, the authors have replaced the original sealing cover with a sterile glove incision protective cover (Figure 2), and is not limited by the hole position of the trocar of the special sealing cover. If necessary, the number of trocars can be increased to insert more instruments or apply $12 \mathrm{~mm}$ Trocar for passing a packing gauze or a big polymer clip in order to better control in case of bleeding. After space formation, three $5 \mathrm{~mm}$ trocars were routinely inserted into the cervical space. The $\mathrm{CO}_{2}$ pressure should be maintained for up to 12 to $14 \mathrm{mmHg}$. Using an energy device, the esophagus was dissected downwards along its surface. The thoracic duct, the azygos vein and the recurrent laryngeal nerve (Figure 3) should be identified and protected, and the subcarinal lymph nodes and the periesophageal lymph nodes were dissected. With mediastinoscopy, the esophagus should be dissected down to the level of the inferior pulmonary vein and even below. In the abdomen, the stomach and the lower esophagus 


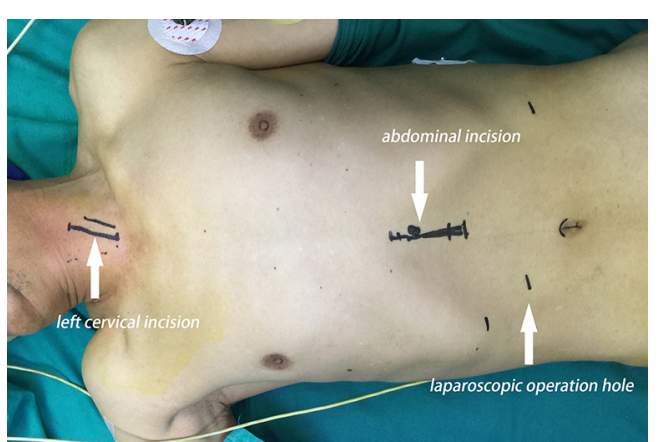

Figure 1 A patient is placed in supine position with planned laparoscopy, small laparotomy, and left cervical incision.

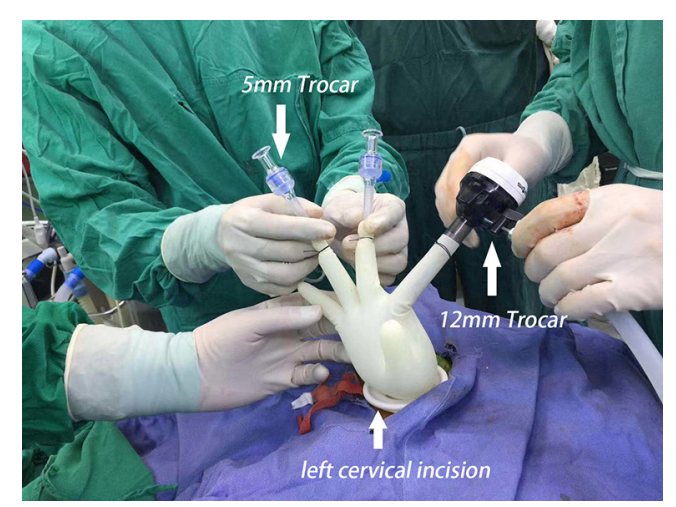

Figure 2 Sterile gloves sealed the protective cover of $5 \mathrm{~cm}$ incision.

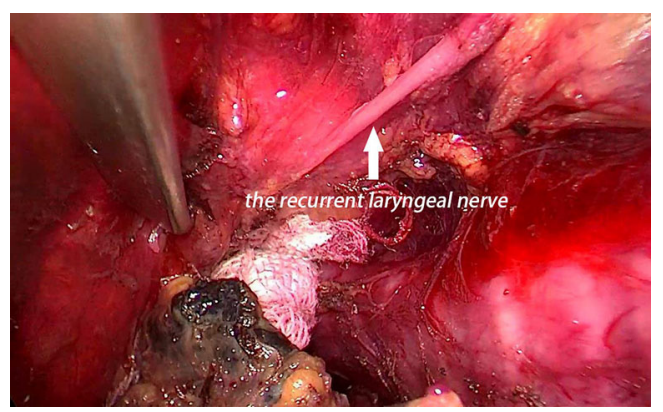

Figure 3 Skeletonization of recurrent laryngeal nerve.

were dissociated under laparoscopy. The laparoscopic dissection through esophageal hiatus meets with the mediastinoscopic dissection, which is close to the level of the inferior pulmonary vein, dissociating the esophagus completely. Meanwhile, the stomach and the perigastric lymph nodes could be dissociated with a laparoscope. The esophagus at the neck was cut off, and the proximal end of the esophagus was prepared for anastomosis, and a traction suture was placed at the distal end of the esophagus to make a gastric conduit. Under the xiphoid process, a $5 \mathrm{~cm}$ midline longitudinal incision was performed. The whole length of the dissociated esophagus and the proximal stomach were pulled out to make a gastric conduit with a width of about $4 \mathrm{~cm}$. Next, the traction suture was used to pull the gastric conduit to perform neck incision, and anastomose the gastric conduit and the proximal end of the esophagus. Indwelling of the jejunal nutrition tube was performed, and sutured each incision to end the operation.

\section{Statistical analysis}

The intraoperative events and management of 60 patients who underwent esophagectomy via transcervical incision inflatable single-port mediastinoscope combined with laparoscopy from March 2017 to January 2020 were retrospectively analyzed. SPSS 22.0 statistical software was used for analysis. Categorical data were expressed as number (percentage).

The study was conducted in accordance with the Declaration of Helsinki (as revised in 2013). The study was approved by Shanghai Changzheng Hospital Ethics Committee [No. CZEC(2018)-06] and individual consent for this retrospective analysis was waived.

\section{Results}

A total of 60 patients underwent esophagectomy via transcervical incision inflatable single-port mediastinoscope combined with laparoscopy, and the tumors were completely resected. No perioperative death and no aortic injury occurred among the patients. Bronchial artery injury and hemorrhage occurred in 2 cases (3.33\%). Bleeding was stopped in a case after compressing for 15 minutes with a gauze strip by introducing a mediastinoscope. In other case, stopping the hemorrhage was not successful with compression; and therefore, the method was converted to video-assisted thoracoscopy, and the bleeding was stopped successfully by suturing the bleeding artery. The bronchial artery combined with azygos vein hemorrhage was observed in a case $(1.67 \%)$. Hemostasis with energy devices and packing remained unsuccessful (Figure 4). Hemorrhage was severe and unable to control through the mediastinoscope. Emergency conversion to thoracotomy was done, and the source of bleeding from the azygos vein that was controlled by clamping and repairing by vascular suturing. Right pleural injury was observed in 3 cases $(5 \%)$, and so pleura 


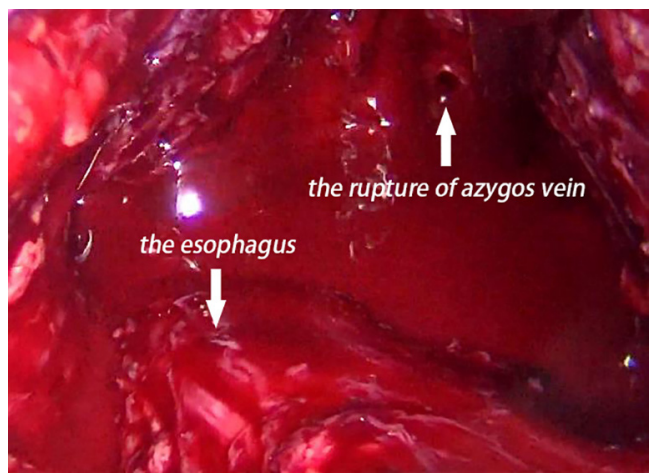

Figure 4 Rupture of azygos vein can be seen under mediastinoscope.

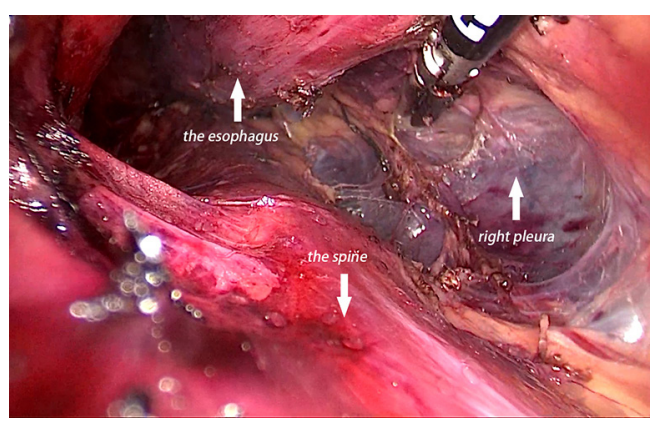

Figure 5 Right pleura can be seen under a mediastinoscope.

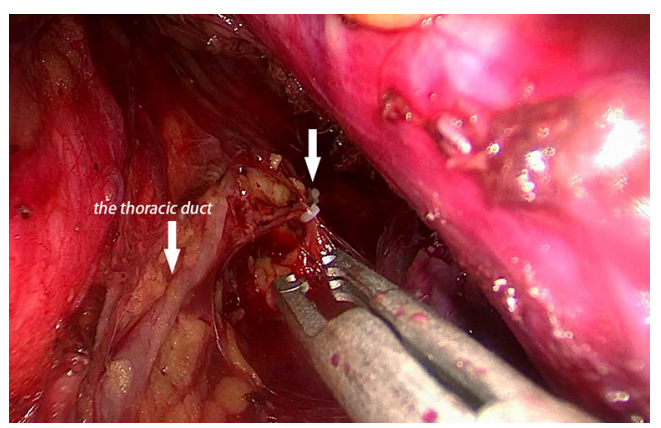

Figure 6 Hemolock clip to close the thoracic duct.

were opened during dissection of the right esophageal wall, and no special treatment was needed (Figure 5). Recurrent laryngeal nerve injury was observed in 7 cases (11.67\%). Hoarseness of voice was noticed post-operatively, and was recovered without any special treatment from 6 months to 1 year after the operation. Thoracic duct injury was observed in 1 case (1.67\%), which led to leakage of lymph in the operative field under mediastinoscopy. The thoracic duct was dissected at T10 and clipped by Hemolock applicator (Figure 6). The patient had no chylothorax after operation. There was no tracheal or bronchial injury in this series.

\section{Discussion}

Esophageal cancer is one of the leading malignancies that is diagnosed and treated by thoracic surgery. But surgical treatment of esophageal cancer is traumatic and is associated with high risk of intraoperative events because of deep anatomical location and adjacent to important structures, such as the aorta and the trachea $(8-10)$. The development of a minimally invasive surgery for esophageal cancer has always been one of the important goals of surgical improvement. Esophagectomy via transcervical incision inflatable single-port mediastinoscope combined with laparoscopy avoids the impact of transthoracic approach on the thorax and significantly reduces surgical trauma when compared with classical procedures such as thoracoscopy combined with laparoscopic esophagectomy. Several studies have reported that compared with transthoracic surgery for esophageal cancer, the incidence of complications and perioperative mortality with singleport inflatable mediastinoscope for esophagectomy showed no significant difference, and is considered as a safe and feasible operation $(5,11-14)$. This study summarized the intraoperative complications and management strategies of 60 cases who underwent esophagectomy via transcervical incision inflatable single-port mediastinoscope combined with laparoscopy. The limited space during inflatable mediastinoscopy might increase the possibility of intraoperative complications, which were higher than that of video-assisted thoracoscopy and thoracotomy, and requires higher skills and experience when intraoperative accidents occur. Operators need to have enough experience and confidence to take decisions to convert to other operations in order to solve these intraoperative complications that cannot be effectively handled by inflatable mediastinoscopy.

Intraoperative bleeding usually occurs due to bronchial artery injury, azygos vein injury, and thoracic aorta injury. Bronchial artery injury is the most common complication that usually occurs during the dissection of the space between the esophagus and the trachea. In most of the cases, the amount of bleeding is small and controllable with energy devices and/or to gauze packing. In cases with more severe bleeding, conversion to thoracoscopy or thoracotomy can be life-saving and should be done immediately when the hemostatic effect remains to be ineffective. Injury of 
the azygos vein remains intense. According to the previous studies, the incidence of azygos vein injury is reported to be about $0.45 \%$ to $0.87 \%$ during open surgery for esophageal cancer $(15,16)$. The high incidence of azygos vein injury in this study might be related to the preliminary clinical treatment of this operation, and is also affected by the inclusion of small sample size. Due to narrow operative space in mediastinoscopy, the surgical field can be easily barred by a small amount of bleeding, making it more difficult to control. Therefore, when dissociating the right wall of the esophagus, the esophagus should be gently pulled towards the left side using a mediastinal retractor to maximize the exposure of the azygos vein and avoid injury. Once the injury to azygos vein occurs, it cannot be controlled without conversion to thoracotomy after gauze packing and compression. The most important is that it is necessary to dissect the azygos vein under direct vision, repair the azygos vein by vascular suture or directly ligate the azygos vein to stop the bleeding. Aortic injury mostly occurs due to excessive heat of energy devices or by tumor invasion. The choice of energy devices is particularly crucial, and safe energy devices with good thermal insulation such as Maryland forceps are considered better for use in these operations. As aortic hemorrhage often leads to disastrous outcomes, it is crucial to evaluate the invasion of the tumor before operation. Such patients might undergo neoadjuvant therapy, and then reassessed the possibility of surgical treatment if tumors are shrinked. When hemorrhage occurs during the operation, then the most important thing for the surgeon is to keep calm, pack a gauze to control the hemorrhage through the mediastinoscope to clean the operative field and gain time to plan. Sterile gloves were used to seal the incision protective cover and replace 5 $\mathrm{mm}$ trocar with $12 \mathrm{~mm}$ trocar during the operation, which assists in achieving gauze packing under mediastinoscope and insert Hemolock clip, and this is of great significance for treating mediastinoscopic bleeding. When large amount of hemorrhage occurs, then it is necessary to immediately convert the operation to thoracotomy. Double lumen intubation is a routine process in these cases as it is necessary to achieve one-lung ventilation in case if conversion is needed.

Pleural injury can occur when dissecting the right side of the esophagus, which can in turn lead to pneumothorax. Because of preoperative intubation and mechanical ventilation, the pulmonary ventilation usually remains unaffected unless a parenchymal injury occurs. Usually, no special treatment is needed for this situation. Some researchers intentionally open the pleura during the procedure for observing any mediastinal bleeding or anastomotic leak during the early postoperative period.

Recurrent laryngeal nerve injury is often difficult to be noticed during the operation, and the utilization of energy devices can easily lead to thermal injury of recurrent laryngeal nerve, which can only be confirmed when the patient has hoarseness after the operation. Therefore, during the procedure, attention should be paid to protect the nerve by careful dissection, identification and thorough knowledge of its anatomy. When dissecting the lymph nodes adjacent to the recurrent laryngeal nerve, then the use of energy devices should be avoided as much as possible, as blunt dissection is preferred in this area. In this series, five patients had intraoperative skeletonization of recurrent laryngeal nerve, and all of these had hoarseness shortly after the surgery. We hypothesized that skeletonization of recurrent laryngeal nerve can easily cause unnecessary injury to the recurrent laryngeal nerve, and so dealing with the nerve during the procedure should be reduced.

Thoracic duct injury is a common complication during esophageal surgeries, in which it can lead to postoperative chylothorax. The incidence of thoracic duct injuries during open surgery for esophageal cancer is about $1.06 \%$ to $1.35 \%(15,16)$. The incidence of thoracic duct injury in this study is $1.67 \%$. Due to the influence of sample size and other reasons, whether the difference between the data is meaningful or not requires further study. The most common place of injury is at T5 level, wherein the duct crosses the midline in front of the vertebral body. The improper use of energy devices and tumor invasion can easily damage the thoracic duct. The branches of thoracic ducts are occasionally injured due to high variation in the anatomy of thoracic duct. Wan Pernis has suggested that the thoracic duct is always present solely at level 8 of the thoracic vertebra to the cisterna chyli, and so when the thoracic duct is suspected to be injured during the operation, then the thoracic duct can be ligated at level 10 of the thoracic vertebra in order to prevent the occurrence of chylothorax (15). A preoperative high-fat diet can effectively fill the thoracic duct to show its course clearly during the procedure, reducing the chance of its damage (13).

Tracheal injury most commonly occurs in cases of external invasion of esophageal cancer. The anatomical layer between the esophagus and the trachea is usually blurred due to external invasion of esophageal cancer, and this can easily lead to tracheal injury when dissecting the 
anterior wall of the esophagus. If preoperative esophageal barium meal examination indicates an ulcerative notch at the anterior wall of the middle esophagus, then fiberoptic bronchoscopy should be performed to determine whether the tracheal membrane is involved or not, and this assists in ruling out the possibility of tumor infiltration into the trachea $(3,15)$. Because of the narrow operating space with the mediastinoscopic technique, it is difficult to repair tracheal injury without conversion to thoracoscopy or thoracotomy. Patients with cancer involving the tracheal membrane should be considered for transthoracic surgery or neoadjuvant therapy. The use of mediastinal retractor and energy devices during operation can also lead to tracheal injury. When using mediastinal retractor to expose the surgical field, the tissue should be pulled and manipulated very gently. During tracheal injury, cooperation with anesthesiologists remains to be crucial. The anesthesiologist should ensure the position and the patency of the doublelumen tube, the sputum and the blood clots in the trachea should be absorbed, and then thoracotomy for repair should be considered.

\section{Conclusions}

As a new surgical method, esophagectomy via transcervical incision inflatable single-port mediastinoscope combined with laparoscopy is considered safe and feasible, but requires improvement when compared with other traditional surgical methods. Due to the influence of surgical space and experience of the surgeons, the incidence of intraoperative complications such as intraoperative bleeding and thoracic duct injury is not dominant as compared with traditional surgical methods. Thoracic surgeons require continuous improvement of their clinical knowledge and skills. Careful preoperative examination and evaluation of the patients, being familiar with the anatomical structure and various methods, wise selection of energy devices and calmly dealing with all kinds of complications assist in making the surgeries successful with fewer intraoperative complications.

\section{Acknowledgments}

Funding: None.

\section{Footnote}

Reporting Checklist: The authors have completed the STROBE reporting checklist. Available at http://dx.doi. org/10.21037/jtd-20-2331

Data Sharing Statement: Available at http://dx.doi. org/10.21037/jtd-20-2331

Conflicts of Interest: All authors have completed the ICMJE uniform disclosure form (available at http://dx.doi. org/10.21037/jtd-20-2331). The authors have no conflicts of interest to declare.

Ethical Statement: The authors are accountable for all aspects of the work in ensuring that questions related to the accuracy or integrity of any part of the work are appropriately investigated and resolved. The study was conducted in accordance with the Declaration of Helsinki (as revised in 2013). The study was approved by Shanghai Changzheng Hospital Ethics Committee [No. CZEC(2018)-06] and individual consent for this retrospective analysis was waived.

Open Access Statement: This is an Open Access article distributed in accordance with the Creative Commons Attribution-NonCommercial-NoDerivs 4.0 International License (CC BY-NC-ND 4.0), which permits the noncommercial replication and distribution of the article with the strict proviso that no changes or edits are made and the original work is properly cited (including links to both the formal publication through the relevant DOI and the license). See: https://creativecommons.org/licenses/by-nc-nd/4.0/.

\section{References}

1. Jie H, Kang S. Current situation of Epidemiology, diagnosis and treatment of esophageal cancer in China and future countermeasures. China Oncology 2011;7:10-3.

2. Huang J, Chen W. Research progress on the application of chemotherapy in multidisciplinary treatment of esophageal cancer. Chinese Journal of Digestion 2013;33:142-4.

3. Wu B, Xu Z, Qiu M, et al. Radical esophagectomy with mediastinoscope for esophageal carcinoma. Chinese Journal of Digestive Surgery 2007;6:407-9.

4. Wu B, Xue L, Qiu M, et al. Video-assisted mediastinoscopic transhiatal esophagectomy combined with laparoscopy for esophageal cancer. J Cardiothorac Surg 2010;5:132.

5. Cao Q, Zhong B, Li X, et al. Single port mediastinoscopic and simultaneous laparoscopic esophagectomy. Chinese Journal of Thoracic and Cardiovascular Surgery 
2019;35:187-9.

6. Fujiwara H, Shiozaki A, Konishi H, et al. Hand-assisted laparoscopic transhiatal esophagectomy with a systematic procedure for en bloc infracarinal lymph node dissection. Dis Esophagus 2016;29:131-8.

7. Fujiwara H, Shiozaki A, Konishi H, et al. Singleport mediastinoscopic lymphadenectomy along the left recurrent laryngeal nerve. Ann Thorac Surg 2015;100:1115-7.

8. Whooley BP, Law S, Murthy SC, et al. Analysis of reduced death and complication rates after esophageal resection. Ann Surg 2001;233:338-44.

9. Jamieson GG, Mathew G, Ludemann R, et al. Postoperative mortality following oesophagectomy and problems in reporting its rate. Br J Surg 2004;91:943-7.

10. Law S, Wong KH, Kwok KF, et al. Predictive factors for postoperative pulmonary complications and mortality after esophagectomy for cancer. Ann Surg 2004;240:791-800.

11. Tan L, Xu Z, Qiu D, et al. On the safety of video-assisted

Cite this article as: Liu C, Chen Z, Wei R, Huang K, Wu B, Xu Z, Fang Y, Ding X, Tang H. Intra-operative events and countermeasures during esophagectomy via transcervical incision inflatable single-port mediastinoscope combined with laparoscopy. J Thorac Dis 2021;13(1):133-139. doi: 10.21037/jtd$20-2331$ mediastinoscopic esophagectomy. Chinese Journal of Minimally Invasive Surgery 2003;3:406-7.

12. Lin J, Iannettoni MD. Transhiatal esophagectomy. Surg Clin North Am 2005;85:593-610.

13. He W, Qiao Z, He J, et al. Application value of single-port inflatable mediastinoscopy combined with laparoscopy in the radical resection of esophageal cancer. Chinese Journal of Digestive Surgery 2018;17:954-8.

14. Fujiwara H, Shiozaki A, Konishi H, et al. Perioperative outcomes of single-port mediastinoscope-assisted transhiatal esophagectomy for thoracic esophageal cancer. Dis Esophagus 2017;30:1-8.

15. Zhang X, Li D, Luo J, et al. Management of accidents during operation of esophageal or cardiac cancer. The Practical Journal of Cancer 2003;18:195-7.

16. Chen W, Wang J, Ren H, et al. Management of accidental complications in the operation of esophageal and cardiac carcinoma. Journal of Xi'an Medical University (Chinese version) 2000;3:265-6. 